\title{
Supercompact cardinals, sets of reals, and weakly homogeneous trees
}

(set theory/descriptive set theory/large cardinals)

\author{
W. HUGH WoodIN
}

Mathematics 253-37, California Institute of Technology, Pasadena, CA 91125

Communicated by Robert M. Solovay, May 12, 1988 (received for review November 7, 1987)

\begin{abstract}
It is shown that if there exists a supercompact cardinal then every set of reals, which is an element of $L(R)$, is the projection of a weakly homogeneous tree. As a consequence of this theorem and recent work of Martin and Steel [Martin, D. A. \& Steel, J. R. (1988) Proc. Natl. Acad. Sci. USA 85, 6582-6586], it follows that (if there is a supercompact cardinal) every set of reals in $L(R)$ is determined.
\end{abstract}

The subtle relationships between the existence of certain large cardinals and regularity properties of various simple sets of reals is one of the striking developments in modern set theory.

One of the first results in this direction is that of R. Solovay (cf. ref. 1), which states that if a measurable cardinal exists then every $\Sigma_{2}^{1}$ set of reals is Lebesgue measurable and has the property of Baire. An unusual aspect of Solovay's argument is the use of the method of forcing. Prior to this the uses of forcing had been limited to obtaining independence results. Martin (2) refined Solovay's result in showing that if there exists a measurable cardinal then every $\Pi_{1}^{1}$ set of reals is determined, briefly $\Pi_{1}^{1}$ determinacy holds. Martin actually proved a slightly stronger result: Suppose for every real, $x$, $x^{*}$ exists. Then $\Pi_{1}^{1}$ determinacy holds. The assertion of $\Pi_{1}^{1}$ determinacy itself implies that every $\Sigma_{2}^{1}$ set of reals is Lebesgue measurable and has the property of Baire. Thus, Martin's theorem may be regarded as a strengthening of Solovay's. The methods Martin used eliminate the need for forcing.

Shelah and Woodin have shown that if a supercompact cardinal exists then every set of reals that belongs to $L(\mathbb{R})$, the smallest inner model of set theory containing the reals and the ordinals, is Lebesgue measurable and has the property of Baire. Since these properties are absolute to $L(\mathbb{R})$, if there exists a supercompact cardinal then the inner model $L(\mathbb{R})$ is a model of set theory without choice (i.e., of Zermelo-Fraenkel) in which every set of reals is Lebesgue measurable and has the property of Baire. The results of Shelah and Woodin were motivated by those of ref. 3.

I show here that the existence of a supercompact cardinal implies that every set of reals that belongs to $L(\mathbb{R})$ has a certain structural representation from which the regularity results, such as measurability, easily follow. This is made more precise through a sequence of definitions.

For our purposes the set of reals, $\mathbb{R}$, is the set $\omega^{\omega}$ of all functions $f: \omega \rightarrow \omega$, where $\omega=\{0,1, \ldots, k, \ldots\}$ is the set of nonnegative integers. We let $\omega^{<\omega}$ denote the set of all finite sequences of elements of $\omega$ and for $s \in \omega^{<\omega}$ let $N_{s}$ be the set, $N_{s}=\left\{f \in \omega^{\omega}: f \nmid l(s)=s\right\}$, where $l(s)=$ length $(s)$. The set $\left\{N_{s}: s \in \omega^{<\omega}\right\}$ generates a topology on $\omega^{\omega}$; it is the product topology derived from the discrete topology on $\omega$. En-

The publication costs of this article were defrayed in part by page charge payment. This article must therefore be hereby marked "advertisement" in accordance with 18 U.S.C. $\$ 1734$ solely to indicate this fact. dowed with this topology $\omega^{\omega}$ is homeomorphic to the Euclidean space of irrationals. Suppose $X$ is a set. We denote by $X^{\omega}$ the set of all functions $f: \omega \rightarrow X$ and we denote by $X^{<\omega}$ the set of all finite sequences of elements of $X$. We adopt the usual convention that $X^{<\omega}$ is the set of all functions $f:$ dom $f \rightarrow X$ such that dom $f \in \omega$ and if $s \in X^{<\omega}$ then $\operatorname{dom} s=l(s)$ $=$ length $(s)$. Suppose $\lambda$ is an ordinal, $\lambda>0$. A tree on $\omega \times \lambda$ is a subset $T \subset \omega^{<\omega} \times \lambda^{<\omega}$ such that for all pairs $(s, t) \in T$, $l(s)=l(t)$ and $(s \mid i, t\lceil i) \in T$ for all $i<l(s), i \in \omega$. Suppose $T$ is a tree on $\omega \times \lambda$. For $s \in \omega^{<\omega}$ and for $x \in \omega^{\omega}$

$$
T_{s}=\left\{t \in \lambda^{<\omega}:(s, t) \in T\right\} \text { and } T_{x}=\bigcup\left\{T_{x} \mid k: k \in \omega\right\}
$$

For each $x \in \omega^{\omega}, T_{x} \subset \lambda^{<\omega}$ and is naturally viewed as a tree on $\lambda .[T]=\left\{(x, f): x \in \omega^{\omega}, f \in \lambda^{\omega}\right.$ and $(x|k, f| k) \in T$ for all $k \in \omega\}$. We also define $p[T]=\{x:(x, f) \in[T]$ for some $f \in$ $\left.\lambda^{\omega}\right\}$. Thus $p[T] \subset \omega^{\omega}$, it is the projection of $T$, and $p[T]=\{x$ $\in \omega^{\omega}: T_{x}$ is not well-founded\}.

Suppose $X$ is a nonempty set. We denote by $m(X)$ the set of countably complete ultrafilters on the Boolean algebra $P(X) . \mu$ is a measure on $X$ if $\mu \in m(X)$. For $\mu \in m(X)$ and $A$ $\subset X$ we write $\mu(A)=1$ to indicate $A \in \mu$. Suppose that $X=$ $Y^{<\omega}$ and that $\mu \in m\left(Y^{<\omega}\right)$. Since $\mu$ is countably additive, there is a unique $k \in \omega$ such that $\mu\left(Y^{k}\right)=1$. Suppose $\mu_{1}, \mu_{2}$ $\in m\left(Y^{<\omega}\right), \mu_{1}\left(Y^{k_{1}}\right)=1$, and $\mu_{2}\left(Y^{k_{2}}\right)=1$. Then $\mu_{1}<\mu_{2}\left(\mu_{2}\right.$ projects to $\left.\mu_{1}\right)$ if $k_{1}<k_{2}$ and, for all $A \subset Y^{k_{1}}, \mu_{1}(A)=1$ if and only if $\mu_{2}\left(A^{*}\right)=1$ where $A^{*}=\left\{s \in Y^{k_{2}}: s \mid k_{1} \in A\right\}$.

For each $\mu \in m(X)$ there is a canonical elementary embedding $j_{\mu}: V \rightarrow M_{\mu}$ of the universe, $V$, into an inner model, $\boldsymbol{M}_{\mu}$, where $M_{\mu}$ is the transitive collapse of $V^{X} / \mu$. Suppose $\mu_{1}, \mu_{2}$ $\in m\left(Y^{<\omega}\right)$ and $\mu_{1}<\mu_{2}$. Then there is also a canonical elementary embedding $j_{\mu_{1} \mu_{2}}: M_{\mu_{1}} \rightarrow M_{\mu_{2}}$ such that $j_{\mu_{2}}=j_{\mu_{1} \mu_{2}}$ 。 $j_{\mu_{1}}$.

Suppose $\left\langle\mu_{k}: k \in \omega\right\rangle$ is a sequence of measures in $m\left(Y^{<\omega}\right)$ such that for each $k \in \omega, \mu_{k}\left(Y^{k}\right)=1$. The sequence $\left\langle\mu_{k}: k \in\right.$ $\omega\}$ is a tower if for all $k_{1}<k_{2}, \mu_{k_{1}}<\mu_{k_{2}}$. The tower, $\left\langle\mu_{k}: k \in\right.$ $\omega\rangle$, is countably complete if for any sequence $\left\langle A_{k}: k \in \omega\right\rangle$ such that for each $k \in \omega, A_{k} \subset Y^{k}$ and $\mu_{k}\left(A_{k}\right)=1$, there exists $f \in Y^{\omega}$ such that $f \nmid k \in A_{k}$ for all $k \in \omega$. A tower of measures in $m\left(Y^{<\omega}\right),\left\langle\mu_{k}: k \in \omega\right\rangle$, is countably complete if and only if the direct limit of the sequence $\left\langle M_{\mu_{k}}: k \in \omega\right\rangle$ under the maps, $j_{\mu_{k_{1}} \mu_{k_{2}}}: M_{\mu_{k_{1}}} \rightarrow M_{\mu_{k_{2}}}$ (where $k_{1}<k_{2}$ ) is wellfounded.

Definition 1: Suppose $\lambda$ is an ordinal, $\lambda>0$. A tree, $T$, on $\omega \times \lambda$ is weakly homogeneous if there is a partial function $\pi$ : $\omega^{<\omega} \times \omega^{<\omega} \rightarrow m\left(\lambda^{<\omega}\right)$ such that

(i) if $(s, t) \in$ dom $\pi$ then $\pi(s, t)\left(T_{s}\right)=1$ and

(ii) for all $x \in \omega^{\omega}, x \in p[T]$ if and only if there exists $y \in \omega^{\omega}$ such that for all $k \in \omega,(x|k, y| k) \in \operatorname{dom} \pi$ and $\langle\pi(x|k, y| k)$ $: k \in \omega\rangle$ is a countably complete tower.

Abbreviations: AD, axiom of determinacy; ZFC, Zermelo-Fraenkel with the axiom of choice. 
Definition 2: Suppose $\lambda$ is an ordinal, $\lambda>0$. A tree, T, on $\omega \times \lambda$ is homogeneous if there is a partial function $\pi: \omega^{<\omega} \rightarrow$ $m\left(\lambda^{<\omega}\right)$ such that

(i) if $s \in \operatorname{dom} \pi$ then $\pi(s)\left(T_{s}\right)=1$ and

(ii) for all $x \in \omega^{\omega}, x \in p[T]$ if and only if for all $k \in \omega, x \mid k$ $\in \operatorname{dom} \pi$, and $\langle\pi(x \uparrow k): k \in \omega\rangle$ is a countably complete tower.

The notions of homogeneous and weakly homogeneous trees arose in the study of descriptive set theory in the context of the axiom of determinacy (AD). The concept of a homogeneous tree is implicit in early work of Martin and was formally isolated by Kechris (4).

Any tree on $\omega \times \omega$ is weakly homogeneous. Thus any $\Sigma_{1}^{1}$ subset of $\omega^{\omega}$ is the projection of a weakly homogeneous tree. Similarly any tree on $\omega \times 1$ is homogeneous and so any closed subset of $\omega^{\omega}$ is the projection of a homogeneous tree.

The primary interest in homogeneous or weakly homogeneous trees exists because of the structural representations they provide for their projections that are sets of reals. Suppose $A \subset \omega^{\omega}$. If $A$ is the projection of a homogeneous tree then $A$ is determined as is any preimage of $A$ via a continuous function $F: \omega^{\omega} \rightarrow \omega^{\omega}$. If $A$ is the projection of a weakly homogeneous tree then enough of the continuous preimages of $\boldsymbol{A}$ are determined so that one can show that many regularity properties hold for $A$-for example, that $A$ is Lebesgue measurable and has the property of Baire.

Notice that if $A \subset \omega^{\omega}$ is the projection of a homogeneous tree and $F: \omega^{\omega} \rightarrow \omega^{\omega}$ is a continuous function then the image of $A$ under $F, B=F^{\prime \prime}(A)$, is the projection of a weakly homogeneous tree. The converse is also true. If $B \subset \omega^{\omega}$ is the projection of a weakly homogeneous tree then there is a continuous function $F: \omega^{\omega} \rightarrow \omega^{\omega}$ and a set $A \subset \omega^{\omega}$ such that $B=$ $F^{\prime \prime}(A)$ and $A$ is the projection of a homogeneous tree.

If there are no measurable cardinals then $A \subset \omega^{\omega}$ is the projection of a homogeneous tree if and only if $A$ is closed. This is essentially because if there are no measurable cardinals then for any $\lambda$ the only elements of $m\left(\lambda^{<\omega}\right)$ are the atomic measures (i.e., principal ultrafilters). Similarly without measurable cardinals $B \subset \omega^{\omega}$ is the projection of a weakly homogeneous tree if and only if $B$ is a $\Sigma_{1}^{1}$ set.

For our purposes an easier formulation of weak homogeneity is actually more relevant. This is given in the easily verified lemma below.

LemMA. Suppose $\lambda$ is an ordinal and that $\mathrm{T}$ is a tree on $\omega$ $\times \lambda$. The tree, $\mathrm{T}$, is weakly homogeneous if and only if there exists a countable set $\sigma \subset \mathrm{m}\left(\lambda^{<\omega}\right)$ such that for all $\mathrm{x} \in \mathrm{p}[\mathrm{T}]$ there is a countably complete tower, $\left\langle\mu_{\mathrm{k}}: \mathrm{k} \in \omega\right\rangle$, of measures in $\sigma$ such that for all $\mathrm{k} \in \omega, \mu_{\mathrm{k}}\left(\mathrm{T}_{\mathrm{x} \mid \mathrm{k}}\right)=1$.

There are two minor points. First, if $T$ is a tree on $\omega \times \lambda$ and $\left\langle\mu_{k}: k \in \omega\right\rangle$ is a countably complete tower of measures in $m\left(\lambda^{<\omega}\right)$, where for some $x \in \omega^{\omega}, \mu_{k}\left(T_{x \mid k}\right)=1$ for each $k \in$ $\omega$, then $x$ is necessarily an element of $p[T]$. The second point is that in the case of weak homogeneity (following the notation in the definition) it is only the range of $\pi$ that is important.

If $\boldsymbol{T}$ is a weakly homogeneous tree then $\sigma$ is a witness for this if $\sigma$ satisfies the conditions in the statement of the lemma. Suppose $\mu$ is a measure in $m(X)$ and that $\kappa$ is an ordinal. The measure $\mu$ is $\kappa$-complete if for any $S \subset \mu$ with $|S|<\kappa, \cap$ $S \in \mu$; i.e., if $S \subset P(X)$ is a set of cardinality $<\kappa$ such that for all $Z \in S, \mu(Z)=1$ then $\mu(\cap\{Z: Z \in S\})=1$. A tree $T$ on $\omega \times \lambda$ is $\kappa$-weakly homogeneous if there exists a witness $\sigma$ for the weak homogeneity of $T$ containing only $\kappa$-complete measures. $T$ is < $\kappa$-weakly homogeneous if $T$ is $\alpha$-weakly homogeneous for each $\alpha>\kappa$.

Martin's proof of $\Pi_{1}^{1}$ determinacy from the existence of a measurable cardinal is in essence a proof of the following. Assume $\kappa$ is a measurable cardinal. Then every $\Pi_{1}^{1}$ set of reals is the projection of a $\kappa$-homogeneous tree. As an immediate consequence, if $\kappa$ is a measurable cardinal then every
$\Sigma_{2}^{1}$ set of reals is the projection of a $\kappa$-weakly homogeneous tree.

THEOREM 1. Suppose $\kappa$ is a supercompact cardinal. Then every set of reals that belongs to $\mathrm{L}(\mathbb{R})$ is the projection of a $\kappa$-weakly homogeneous tree.

\section{Proof of Theorem 1}

An essential ingredient in the proof of Theorem 1 is the use of generic elementary embeddings. We develop some of the necessary machinery. We use standard notation. For example, $X^{Y}=\{f \mid f: Y \rightarrow X\}$. If $f: Y \rightarrow X$ and $Z \subset Y$ then $f^{\prime \prime}(Z)$ $=\{f(t): t \in Z\}$. When considering an elementary embedding $j: V \rightarrow M$ it is always assumed that $j$ is an elementary embedding from $(V, \varepsilon)$ into $(M, \varepsilon)$ and that $M$ is transitive. If we write $j: V \rightarrow(M, E)$ then nothing is implicitly assumed except that $j$ is an elementary embedding from $(V, \varepsilon)$ into $(M$, $E)$; in particular, $M$ need not be transitive. Suppose $j: V \rightarrow$ $M$ is an elementary embedding. Then the critical point of $j$, denoted $\operatorname{cp}(j)$, is the least ordinal $\alpha$, if it exists, such that $j(\alpha)$ $\neq \alpha$. Suppose $X$ is a transitive set. Recall that $\kappa$ is $X$-supercompact if there exists an elementary embedding $j: V \rightarrow M$ such that $\kappa=\operatorname{cp}(j), M^{X} \subset M$ and $X \in j\left(V_{\kappa}\right) . \kappa$ is supercompact if $\kappa$ is $X$-supercompact for each $X$.

Definition 3: (i) A nonempty set $b$ is stationary if for any function $F:(\cup b)^{<\omega} \rightarrow \cup b, F^{\prime \prime}\left(a^{<\omega}\right) \subset a$ for some $a \in b$;

(ii) a set $c$ is closed if for some function $F:(\cup c)^{<\omega} \rightarrow \cup c$, $c=\left\{a \subset \cup c: F^{\prime \prime}\left(a^{<\omega}\right) \subset a\right\}$

(iii) a set $a$ is closed and unbounded in $b(b \neq \emptyset)$ if $a=c \cap$ $b$ for some closed set $c$ with $\cup c=\cup b$;

(iv) a set $a \subset b$ is stationary in $b$ if $a$ is stationary and $\cup a=$ Ub.

Notice that if $\cup a \in a$ then $a$ is stationary (these are the degenerate stationary sets). There is some conflict with standard usage: a cofinal subset of a singular limit ordinal is never stationary in the preceding sense.

Lemmas 1 and 2 are easy consequences of the definition. A function $f: b \rightarrow \cup b$ is a choice function if for all $A \in b$, if $A \neq \emptyset$, then $f(A) \in A$.

LemMA 1. Suppose $\mathrm{x} \subset \cup \mathrm{b}$ and $\mathrm{b}$ is stationary. Then $\{\mathrm{A} \cap$ $\mathrm{x}: \mathrm{A} \in \mathrm{b}\}$ is stationary.

LEMMA 2. Suppose $\mathrm{b}$ is stationary and $\mathrm{f}: \mathrm{b} \rightarrow \cup \mathrm{b}$ is $a$ choice function. Then for some set a stationary in $\mathrm{b}, \mathrm{f}\lceil\mathrm{a}$ is constant.

Suppose $a$ is a set. Let $h t(a)=\sup \{r k(x): x \in a\}=$ least $\alpha$ such that $\cup a \subset V_{\alpha}$.

For each ordinal $\alpha$ define a partial order $\mathbb{P}_{<\alpha}$ as follows: $\mathbb{P}_{<\alpha}=\{a: a$ is stationary and $h t(a)<\alpha\}$ and for all $a, b \in$ $\mathbb{P}_{<\alpha}, a \leq b$ if $(i) \cup b \subset \cup a$ and $(i i)$ for each $Z \in a, Z \cap(\cup b) \in$ b. $\mathbb{Q}_{<\alpha}$ is the suborder of $\mathbb{P}_{<\alpha}$ given by $\mathbb{Q}_{<\alpha}=\left\{a \in \mathbb{P}_{<\alpha}: a \subset\right.$ $\left.P_{\omega_{1}}(\cup a)\right\}=\left\{a \in \mathbb{P}_{<\alpha}\right.$ : each $Z \in a$ is countable $\}$.

The following definition is of central importance. A set $X$ end-extends $Y$ if $Y \subset X$ and for all $Z \in Y, Z \cap Y=Z \cap X$.

Definition 4: Suppose $\delta$ is an inaccessible cardinal. $A \subset$ $\mathbb{P}_{<\delta}$ is semiproper in $\mathbb{P}_{<\delta}$ if

$\operatorname{sp}(A)=\left\{X \subset V_{\delta+1}:\right.$ For some $Y<V_{\delta+1}, X \subset Y, Y$ end-

extends $X \cap V_{\delta}$ and $Y \cap(\cup a) \in a$ for some $\left.a \in A \cap Y\right\}$

contains a set closed and unbounded in $P_{\delta}\left(V_{\delta+1}\right)$.

Similarly, $A \subset \mathbb{Q}_{<\delta}$ is semiproper in $\mathbb{Q}_{<\delta}$ if $\operatorname{sp}(A)$ contains a set closed and unbounded in $P_{\omega_{1}}\left(V_{\delta+1}\right)$.

We shall restrict our attention to $\mathbb{Q}_{<\delta}$ though much of what we prove holds in an analogous form for $\mathbb{P}_{<\delta}$. Suppose $A \subset$ $Q_{<\delta}$ and let $B=P_{\omega_{1}}\left(V_{\delta+1}\right) \backslash \operatorname{sp}(A)$. Then $A$ is not semiproper if and only if $B$ is stationary in $P_{\omega_{1}}\left(V_{\delta+1}\right)$. One can show that if $A \subset \mathbb{Q}_{<\delta}$ is semiproper then $A$ is predense in $\mathbb{Q}_{<\delta}-$ i.e., $\{a$ $\in \mathbb{Q}_{<\delta}: a \leq b$ for some $\left.b \in A\right\}$ is dense in $\mathbb{Q}_{<\delta}$.

LEMMA 3. Suppose $\mathrm{M}$ is a transitive set, $\mathrm{M}^{\mathrm{V}^{\circ}} \subset \mathrm{M}$ and $\mathrm{F} \in$ 
$\mathrm{M}$ for all $\mathrm{F}: \mathrm{V}_{\delta+1}^{<\omega} \rightarrow \mathrm{V}_{\delta+1}$. Suppose $\mathrm{A} \subset \mathbb{Q}_{<\delta}$. The following are equivalent.

(i) A is semiproper in $\mathbb{Q}_{<\delta}$.

(ii) For any $\mathrm{X}<\mathrm{M}$ with $\mathrm{A}, \delta \in \mathrm{X}$ and $|\mathrm{X}|<\omega_{1}$, there exists $\mathrm{Y}<\mathrm{M}$ such that $\mathrm{X} \subset \mathrm{Y}, \mathrm{Y}$ end-extends $\mathrm{X} \cap \mathrm{V}_{\delta}$ and $\mathrm{Y} \cap$ (Ua) $\in$ a for some a $\in Y \cap A$.

(iii) For any $\mathrm{X}<\mathrm{M}$ with $\mathrm{A}, \delta \in \mathrm{X}$ and $|\mathrm{X}|<\omega_{1}$, there exists $\mathrm{Y} \subset \mathrm{V}_{\delta}$ such that $\mathrm{Y}$ end-extends $\mathrm{X} \cap \mathrm{V}_{\delta}, \mathrm{Y} \cap(\mathrm{Ua}) \in \mathrm{a}$ for some $\mathrm{a} \in \mathrm{Y} \cap \mathrm{A}$, and $\mathrm{f}^{\prime \prime}\left(\mathrm{Y}^{<\omega}\right) \subset \mathrm{Y}$ for all $\mathrm{f}: \mathrm{V}_{\delta}^{<\omega} \rightarrow \mathrm{V}_{\delta}$, $\mathbf{f} \in \mathbf{X}$.

Proof: We shall only use this lemma in the case $M \vDash$ Zermelo-Fraenkel, with the axiom of choice (ZFC). Clearly statement $i i$ of Lemma $3 \rightarrow$ statement $i$. To see that $i i i \rightarrow i i$ observe that since $M^{V_{\delta}} \subset M$ it follows that if $X<M$ and if $Y$ $\subset V_{\delta}$ then $Y^{*}<M$, where $Y^{*}=\left\{f(s): s \in Y^{<\omega}\right.$ and $\left.f \in X\right\}$. To see that $i \rightarrow i i$ suppose $A \subset \mathbb{Q}_{<\delta}$ is semiproper, $X<M, X$ is countable and $A \in X$. Since $X<M$ there exists $F: V_{\delta+1}^{<\omega}$ $\rightarrow V_{\delta+1}, F \in X$, such that $c_{F} \cap P_{\omega_{1}}\left(V_{\delta+1}\right) \subseteq \operatorname{sp}(A)$, where $c_{F}$ $=\left\{Y \subset V_{\delta+1}: F^{\prime \prime}\left(Y^{<\omega}\right) \subset Y\right\}$. Clearly $\left.F^{\prime \prime}\left((X) \cap V_{\delta+1}\right)^{<\omega}\right) \subset X$ and so $X \cap V_{\delta+1} \in \operatorname{sp}(A)$. Choose $Y<V_{\delta+1}$ such that $X \cap$ $V_{\delta+1} \subset Y, Y$ end-extends $X \cap V_{\delta}$ and $Y \cap(\cup a) \in a$ for some $a \in Y \cap A$. Let

$$
Y^{*}=\left\{f(s): f \in X \text { and } s \in\left(Y \cap V_{\delta}\right)^{<\omega}\right\}
$$

Then $Y^{*} \cap V_{\delta}=Y \cap V_{\delta}, X \subset Y^{*}$ and $Y^{*}<M$. This proves statement $i i$ assuming $i$. Finally $i i \rightarrow$ iii is trivial.

THEOREM 2. Suppose $\kappa$ is $\mathrm{V}_{\kappa+1}$-supercompact and that $\mathbf{A}$ $\subset \mathbb{Q}_{<\kappa}$ is predense. Then $\mathrm{A}$ is semiproper in $\mathbb{Q}_{<\kappa}$.

Proof: Fix an elementary embedding $j: V \rightarrow M$ such that $\operatorname{cp}(j)=\kappa$ and $M^{V_{\kappa+1}} \subset M$. Suppose $A \subset \mathbb{Q}_{<\kappa}$ is predense. Let $B=P_{\omega_{1}}\left(V_{\kappa+1}\right) \backslash \operatorname{sp}(A)$. Assume toward a contradiction that $A$ is not semiproper in $\mathbb{Q}_{<\kappa}$. Hence $B$ is stationary in $P_{\omega_{1}}\left(V_{\kappa+1}\right) . M^{V_{\kappa+1}} \subset M$ and so $B \in M$. Thus $M \vDash$ " $B$ is stationary" and so $B \in j\left(\mathbb{Q}_{<\kappa}\right)$. Choose $a \in j(A)$ and $b \in j\left(\mathbb{Q}_{<\kappa}\right)$ such that $b \leq a$ and $b \leq B$. Let $\lambda=j(\kappa+4)$. Choose $Z<M_{\lambda}$ $=V_{\lambda} \cap M$ such that $\left\{j \mid V_{\kappa+1}, A, a, b\right\} \subset Z, Z \cap(\cup b) \in b$ and $Z \in M$. Since $M \vDash$ " $b$ is stationary" and $M^{V_{\kappa+1}} \subset M, Z$ exists. Let $Z_{0}=Z \cap V_{\kappa+1}$. Since $b \leq B$ in $j\left(Q_{<\kappa}\right), Z_{0} \in B$. Therefore $j\left(Z_{0}\right) \in j(B)$. But $Z_{0}$ is countable, hence $j\left(Z_{0}\right)=$ $j^{\prime \prime}\left(Z_{0}\right)$. But then $Z \cap j\left(V_{\kappa+1}\right)$ end-extends $j\left(Z_{0}\right) \cap j\left(V_{\kappa}\right)$ since $j\left(Z_{0}\right) \cap j\left(V_{\kappa}\right)=\left(j^{\prime \prime}\left(Z_{0}\right)\right) \cap j\left(V_{\kappa}\right)=Z_{0} \cap V_{\kappa}=Z \cap V_{\kappa}$. Finally $Z \cap(\cup a) \in a$ and $a \in j(A) \cap Z$. Thus $M \vDash " Z \cap j\left(V_{k+1}\right)$ is a witness that $j\left(Z_{0}\right) \in j(\operatorname{sp}(A))$." Therefore, $j\left(Z_{0}\right) \in j(\operatorname{sp}(A))$ and so $Z_{0} \in \operatorname{sp}(A)$ contradicting $Z_{0} \in B$ since $B=P_{\omega_{1}}\left(V_{\kappa+1}\right) \backslash$ $\operatorname{sp}(A)$.

COROllary. Suppose $\kappa$ is $\mathrm{V}_{\kappa+1}$-supercompact. Then $\{\delta: \delta$ $<\kappa, \delta$ is inaccessible, and each predense $A \subset \mathbb{Q}_{<\delta}$ is semiproper in $\left.\mathbb{Q}_{<\delta}\right\}$ is stationary in $\kappa$.

Proof: Let $j: V \rightarrow M$ be an elementary embedding with $\operatorname{cp}(j)=\kappa$ and $V_{\kappa+2} \subset M$. By Theorem 2 and since $V_{\kappa+2} \subset M$, $M \vDash$ "each predense $A \subset \mathbb{Q}_{<\kappa}$ is semiproper in $\mathbb{Q}_{<\kappa}$." The corollary follows.

THEOREM 3. Suppose $\kappa$ is $\mathbf{V}_{\kappa+1}$-supercompact and that $\mathbf{G}$ $\subset \mathbf{Q}_{<\kappa}$ is $\mathrm{V}$-generic. Then there is a generic elementary embedding $\mathrm{j}: \mathrm{V} \rightarrow \mathrm{M} \subset \mathrm{V}[\mathrm{G}]$ such that $\mathrm{M}^{<\kappa} \subset \mathrm{M}$ in $\mathrm{V}[\mathrm{G}]$ and for each $a \in G, j^{\prime \prime}(\cup a) \in j(a)$.

Proof: For $a \in \mathbb{Q}_{<\kappa}$ let $N S_{a} \subset P(a)$ be the ideal $N S_{a}=\{b$ $C a: b$ is not stationary in $a\}$. We work in $V[G]$ except that for $a \in G ; P(a), N S_{a}$ and $V^{a}$ are as computed in $V$. Thus for $a \in G, U_{a}=P(a) \cap G$ defines a $V$-ultrafilter on $P(a) / N S_{a}$. This gives an elementary embedding $j_{a}: V \rightarrow\left(N_{a}, E_{a}\right) \sim$ $V^{a} / U_{a}$. Suppose $a \in G, b \in G$ and $\cup a \subset \cup b$. Then there is a canonical elementary embedding $j_{a b}:\left(N_{a}, E_{a}\right) \rightarrow\left(N_{b}, E_{b}\right)$ such that $j_{b}=j_{a b} \circ j_{a}$. Taking the direct limit over $a$ in $G$ yields $j: V \rightarrow(N, E)$. For $a \in G$ and $f \in V^{a}$ let $[f]$ denote the element $f$ defines in $N$. Hence if $f, g \in V^{a},[f] E[g]$ if and only if for some $b \in G, b \leq a$ and $f(Y \cap(\cup a)) \in g(Y \cap(\cup a))$ for all $Y \in b$. Clearly $N=\left\{[f]: f \in V^{a}\right.$ for some $\left.a \in G\right\}$. Fix $a \in G$ and let $e_{a}: a \rightarrow a$ be the identity function. Clearly [ $\left.e_{a}\right]$ $E j(a)$. I claim that $\left[e_{a}\right]=j^{\prime \prime}(\cup a)$ in the sense that for all $c \in$ $N, c E\left[e_{a}\right]$ iff $c=j(X)$ for some $X \in \cup a$. To see this, fix $c \in$ $N$ such that $c E\left[e_{a}\right]$. Choose $b \in G$ and $g \in V^{b}$ such that $b \leq$ $a, c=[g]$, and $g(Y) \in Y \cap(\cup a)$ for all $Y \in b$. Hence $g$ is a choice function and so by genericity there exists $b^{*} \in G$ such that $b^{*} \leq b$ and $g\left\{\left\{Y \cap(\cup b): Y \in b^{*}\right\}\right.$ is constant. Take for $X$, this constant value. We now prove that $(N, E)$ is wellfounded and that its transitive collapse, $M$, is closed under $<$ $\kappa$ sequences in $V[G]$. This is equivalent to showing that if $X$ $\subset N$ and $|X|<\kappa$ then for some $Z \in N, X=\{t: t E Z\}$. Suppose $\left\langle\tau_{\alpha}: \alpha<\lambda\right\rangle$ is a $\lambda$ sequence of terms for elements of $N$ where $\lambda<\kappa$ and the sequence is in $V$. Fix $a_{0} \in G$ and we now work in $V$. Assume that for $\alpha<\lambda$, [[ $\left.\left.\tau_{\alpha} \in N\right]\right]=1$. For each $\alpha<\lambda$ fix a maximal antichain $A_{\alpha} \subset \mathbb{Q}_{<\kappa}$ and a function $F_{\alpha}: A_{\alpha} \rightarrow V$ such that for $b \in A_{\alpha}, F_{\alpha}(b) \in V^{b}$ and $b$ 蜘 $\tau_{\alpha}=$ $\left[F_{\alpha}(b)\right]$." By Corollary to Theorem 2 there is an inaccessible cardinal $\delta<\kappa$ such that $\delta>\lambda, \delta>h t\left(a_{0}\right)$ and such that $A_{\alpha} \cap$ $V_{\delta}$ is semiproper in $\mathbb{Q}_{<\delta}$ for each $\alpha<\lambda$. Let $a=\left\{X \in P_{\omega_{1}}\left(V_{\delta}\right)\right.$ $: X<V_{\delta}$ and for each $\alpha \in X \cap \lambda, X \cap(\cup b) \in b$ for some $b \in$ $\left.\boldsymbol{X} \cap \boldsymbol{A}_{\alpha}\right\}$.

Claim. $a_{0}^{*}=\left\{X \in a: X \cap\left(\cup a_{0}\right) \in a_{0}\right\}$ is stationary in $\mathbf{P}_{\omega_{1}}\left(\mathrm{~V}_{\delta}\right)$.

Proof of Claim: Suppose $H: V_{\delta}^{<\omega} \rightarrow V_{\delta}$. We find $X \in a_{0}^{*}$ such that $H^{\prime \prime}\left(X^{<\omega}\right) \subset X$. Choose $X_{0} \in P_{\omega_{1}}\left(V_{\kappa}\right)$ such that $X_{0}<$ $V_{\kappa}, X_{0} \cap\left(\cup a_{0}\right) \in a_{0}$ and $\left\{H, a_{0},\left\langle A_{\alpha}: \alpha<\lambda\right\rangle\right\} \subset X_{0}$. Since $a_{0}$ is stationary $X_{0}$ exists. Choose an elementary chain $\left\langle X_{\gamma}: \gamma \in\right.$ $\left.X_{0} \cap \lambda\right\rangle$, starting with $X_{0}$, such that (i) for all $\gamma \in X_{0} \cap \lambda, X_{\gamma}$ $<V_{\kappa},\left|X_{\gamma}\right|=\omega$ and $X_{\gamma+1} \cap(\cup b) \in b$ for some $b \in A_{\gamma} \cap X_{\gamma+1}$ $\cap V_{\delta}$ and (ii) for all $\gamma_{1}<\gamma_{2}$, if $\left\{\gamma_{1}, \gamma_{2}\right\} \subset X_{0} \cap \lambda$ then $X_{\gamma_{2}}$ endextends $X_{\gamma_{1}} \cap V_{\delta}$. Using part ii of Lemma 3, the chain is easily constructed. Let $X=\cup\left\{X_{\gamma} \cap V_{\delta}: \gamma \in X_{0} \cap \lambda\right\}$. Note $\lambda$ $<\delta$ and $\lambda \in X_{0}$. Hence $X \cap \lambda=X_{0} \cap \lambda$ and so $X \in a_{0}^{*}$ and $H^{\prime \prime}\left(X^{<\omega}\right) \subset X$. $\square$ proof of claim

For each $\alpha<\lambda$ if $X<V_{\delta}$ then $\left\{b: b \in A_{\alpha} \cap X\right.$ and $X \cap$ $(\cup b) \in b\}$ contains at most one element. This is because $A_{\alpha}$ is an antichain. Define $f \in V^{a \dot{0}}$ by $f(Y)=\left\{F_{\gamma}(b)(Y \cap(\cup b))\right.$ : $\gamma \in Y \cap \lambda, b \in Y \cap A_{\gamma} \cap V_{\delta}$ and $\left.Y \cap(\cup b) \in b\right\}$. Thus $a_{0}^{*}$ it " $\{c \in N \mid c E[f]\}=\left\{\tau_{\alpha}: \alpha<\lambda\right\}$." By genericity we can choose $a_{0}$ so that $a_{0}^{*} \in G$.

Henceforth an inner model is a transitive class, possibly a set, closed under the primitive recursive set functions. If $M$ is an inner model then $M(X)$ is the smallest inner model containing $M \cup\{X\}$. For ordinals, $\alpha$, let $\operatorname{Coll}(\omega,<\alpha)$ be the partial order of finite conditions for the Levy collapse of ordinals $<\alpha$ to $\omega$. Suppose $M$ is an inner model of ZFC, $\alpha \in$ $M$, and $G \subset \operatorname{Coll}(\omega,<\alpha)$ is $M$-generic. Let $\tau=\cup\{M(G \cap$ Coll $(\omega,<\beta)) \cap \mathbb{P}: \beta<\alpha\}$ and let $N_{G}=M(\tau)$. Standard arguments show that if $G_{1}$ and $G_{2}$ are each $M$-generic for Coll $(\omega,<\alpha)$ then $N_{G_{1}} \equiv_{M} N_{G_{2}}$; i.e., $N_{G_{1}}$ and $N_{G_{2}}$ satisfy the same formulas of the language of set theory with parameters from $M$. We say an inner model, $N$, is a symmetric extension of $M$ for Coll $(\omega,<\alpha)$ if $M \subset N$ and $N \equiv_{M} N_{G}$ in $V[G]$, where $G \subset \operatorname{Coll}(\omega,<\alpha)$ is $V$-generic. This is a first-order property of $N$ in a predicate for $M$.

LEMMA 4. Suppose $M$ is an inner model of $Z F C, \lambda \in M$ and $\mathrm{M} \vDash$ " $\lambda$ is a strong limit cardinal." Suppose $\tau \subset \mathbb{R}$ is such that (i) for all $\mathrm{x} \in \tau$, there exits $\mathbb{P} \in \mathbf{M}$ and $\mathrm{g} \subset \mathbb{P}$ such that $\mathrm{M} \vDash "|\mathbb{P}|<\lambda$," $\mathrm{g}$ is $\mathrm{M}$-generic for $\mathbb{P}$, and $\mathrm{x} \in \mathrm{M}[\mathrm{g}]$, (ii) for all $\mathrm{x}, \mathrm{y} \in \tau, \mathbb{R} \cap \mathrm{M}(\mathrm{x}, \mathrm{y}) \subset \tau$, and (iii) $\sup \left\{\omega_{1}^{\mathrm{M}(\mathrm{x})}: \mathrm{x} \in \tau\right\}$ $=\lambda$. Then $\mathbb{R} \cap \mathbf{M}(\tau)=\tau$ and $\mathrm{M}(\tau)$ is a symmetric extension of $\mathbf{M}$ for $\operatorname{Coll}(\omega,<\lambda)$.

THEOREM 4. Suppose $\kappa$ is supercompact and $\mathrm{G} \subset \mathbb{Q}_{<\kappa}$ is $\mathrm{V}$-generic. Then $\left\{\delta: \delta<\kappa\right.$ and $\mathrm{G} \cap \mathbb{Q}_{<\delta}$ is $\mathrm{V}$-generic for $\left.Q_{<\delta}\right\}$ is unbounded in $\kappa$.

Proof: We work in $V$. Fix $a_{0} \in \mathbb{Q}_{<\kappa}$. By Corollary to Theorem 2 there exists $\delta>h t\left(a_{0}\right)$ such that $\delta$ is inaccessible, $\delta<$ $\kappa$, and each dense $A \subset \mathbb{Q}_{<\delta}$ is semiproper. Let $a_{0}^{*}=\{X \in$ $P_{\omega}\left(V_{\delta+1}\right): X \cap\left(\cup a_{0}\right) \in a_{0}$ and for each dense $A \subset Q_{<\delta}$ if $A$ $\in X$ then $X \cap(\cup b) \in b$ for some $b \in X \cap A\}$. By an elemen- 
tary chain argument, using Lemma 3, as above (Proof of Theorem 3), $a_{0}^{*}$ is stationary. It follows that $a_{0}^{*} \leq a_{0}$ and $a_{0}^{*}$ It " $G \cap \mathbb{Q}_{<\delta}$ is $V$-generic."

Corollary. Suppose $\kappa$ is supercompact and $\mathbf{G} \subset \mathbb{Q}_{<x}$ is $\mathrm{V}$-generic. Let $\tau=(\mathbb{R})^{\mathrm{V}[\mathrm{G}]}$. Then $\mathrm{V}(\tau)$ is a symmetric extension of $\mathrm{V}$ for $\operatorname{Coll}(\omega,<\kappa)$.

Proof: Suppose $\tau \in V^{\mathbf{Q}<\kappa}$ is a term, $a \in \mathbb{Q}_{<\kappa}$, and $a$ It " $\tau \subset$ $\omega$." Then there exists $\tau^{*} \in V^{\mathbf{Q}<\kappa} \cap V_{\kappa}$ and $b \leq a$ such that $b$ It " $\tau=\tau^{*}$." Given this it follows by Theorem 4 that for each $x \subset \omega$ with $x \in V[G]$, there exists $\delta<\kappa$ such that $x \in V[G \cap$ $\mathbb{Q}_{<\delta}$ ] and $G \cap \mathbb{Q}_{<\delta}$ is $V$-generic for $\mathbb{Q}_{<\delta}$. The corollary now follows by Lemma 4 provided $\kappa=\omega_{1}[\{\dot{G}]$. By Theorem 3, $\kappa$ $\leq \omega_{1}^{V[G]}$. This combined with the preceding shows $\kappa=$ $\omega_{1}^{\text {VIG]. }}$

To see that $b$ and $\tau^{*}$ exist define for each $i<\omega$ a set $A_{i} \subset$ $\mathbb{Q}_{<\kappa}$ by $A_{i}=\{c \leq a: c$ It " $i \in \tau$ " or $c$ If " $i \notin \tau$ " $\}$. Thus $A_{i}$ is dense below $a$. Choose $b \leq a$, as above, such that for all $Z \in$ $b$ and for all $i<\omega, Z \cap(\cup c) \in c$ for some $c \in A_{i} \cap b . \tau^{*}$ is easily defined from $b$.

Recall $\mathbb{R}^{*}$ is the theory of $L(\mathbb{R})$ in parameters from $\mathbb{R} \cup\left\{\gamma_{k}\right.$ $: k \in \omega\}$, where $\left\langle\gamma_{k}: k \in \omega\right\rangle$ is any increasing sequence of Silver indiscernibles for $L(\mathbb{R})$ see (ref. 5$)$. We uniformly view $\mathbb{R}^{*} \subset \mathbb{R}$. Suppose $\mathbb{R}^{*}$ exists, $M$ is an inner model of $Z F C$ and that $M \vDash$ " $\mathbb{R}^{*}$ exists." Then $(\mathbb{R})^{M},\left(\mathbb{R}^{*}\right)^{M}$ are as computed in $M$. Of course $(\mathbb{R})^{M} \subset \mathbb{R}$ but if $\left(\mathbb{R}^{*}\right)^{M} \subset \mathbb{R}^{*}$ then $(L(\mathbb{R}))^{M} \equiv$ $L(\mathbb{R})$ in a very strong sense.

THEOREM 5. Suppose $\kappa$ is supercompact and suppose $\mathrm{V}(\tau)$ is a symmetric extension of $\mathrm{V}$ for $\operatorname{Coll}(\omega,<\kappa)$. Then $\left(\mathbb{R}^{*}\right)^{\mathrm{V}} \subset$ $\left(\mathbb{R}^{*}\right)^{\mathrm{V}(\tau)}$.

Proof: Suppose $G \subset \mathbb{Q}_{<\kappa}$ is $V$-generic and let $j: V \rightarrow M \subseteq$ $V[G]$ be the induced embedding. Let $\tau_{G}=(\mathbb{R}) V[G]$. Thus $V\left(\tau_{G}\right)$ is a symmetric extension of $V$ for $\operatorname{Coll}(\omega,<\kappa)$. Clearly $\left(\mathbb{R}^{*}\right) \subset\left(\mathbb{R}^{*}\right)^{M}$. Since $M^{\omega} \subset M$ in $V[G],\left(\mathbb{R}^{*}\right)^{M}=\left(\mathbb{R}^{*}\right)^{V[G]}=$ $\left(\mathbb{R}^{*}\right)^{V\left(\tau_{G}\right)}$. So $\left(\mathbb{R}^{*}\right)^{V} \subset\left(\mathbb{R}^{*}\right)^{V\left(\tau_{G}\right)}$ and therefore by homogeneity $\left(\mathbb{R}^{*}\right)^{V} \subset\left(\mathbb{R}^{*}\right)^{V(\tau)}$.

THEOREM 6. Suppose $\kappa$ is supercompact. Suppose $\mathbb{P}_{1}$ is a partial order and $G_{1} \subset \mathbb{P}_{1}$ is $V$-generic. Suppose $\mathbb{P}_{2} \in V\left[G_{1}\right]$ is a partial order and $\mathrm{G}_{2} \subset \mathbb{P}_{2}$ is $\mathrm{V}\left[\mathrm{G}_{1}\right]$-generic. Then $\left(\mathbb{R}^{*}\right)^{\mathrm{V}\left[\mathrm{G}_{1}\right]}$ $\subset\left(\mathbb{R}^{*}\right)^{\mathbf{V}\left[\mathbf{G}_{1}\right]\left[\mathbf{G}_{2}\right]}$.

Proof: By reflection ( $\kappa$ is supercompact) we can reduce to the case $\mathbb{P}_{1} \in V_{\kappa}$ and $\mathbb{P}_{2} \in V_{\kappa}\left[G_{1}\right]$. Hence $V\left[G_{1}\right] \vDash$ " $\kappa$ is supercompact" and $V\left[G_{1}\right]\left[G_{2}\right] \vDash$ " $\kappa$ is supercompact." Let $g$ $\subset \operatorname{Coll}(\omega,<\kappa)$ be $V\left[G_{1}\right]\left[G_{2}\right]$-generic and let $\tau=$ $(\mathbb{R})^{V\left[G_{1}\right]\left[G_{2}\right][g]} . \mathbb{P}_{1} \in \mathrm{V}_{\kappa}$ and $\mathbb{P}_{2} \in \mathrm{V}_{\kappa}\left[G_{1}\right]$ and so by Lemma 4 , $V(\tau)$ is a symmetric extension of both $V\left[G_{1}\right]$ and $V\left[G_{1}\right]\left[G_{2}\right]$ for Coll $(\omega,<\kappa)$. Hence by Theorem $5,\left(\mathbb{R}^{*}\right)^{\left.V G_{1}\right]} \subset\left(\mathbb{R}^{*}\right)^{V(\tau)}$ and $\left(\mathbb{R}^{*}\right)^{V\left[G_{1}\right]\left[G_{2}\right]} \subset\left(\mathbb{R}^{*}\right)^{V(\tau)}$. Hence $\left(\mathbb{R}^{*}\right)^{V\left[\hat{G}_{1}\right]} \subset\left(\mathbb{R}^{*}\right)^{V\left(G_{1}\right]\left[G_{2}\right]}$.

THEOREM 7. Suppose $\kappa$ is supercompact. There are trees $T$, $\mathrm{T}^{*}$ on $\omega \times \kappa$ such that for any partial order $\mathbb{P} \in \mathrm{V}_{\kappa}$, if $\mathrm{G} \subset \mathbb{P}$ is $\mathrm{V}$-generic then $\mathrm{V}[\mathrm{G}] \vDash " \mathrm{p}[\mathrm{T}]=\mathbb{R}^{*}$ and $\mathrm{p}\left[\mathrm{T}^{*}\right]=\omega^{\omega} \backslash$ $\mathrm{p}[\mathrm{T}]$."

Proof: Suppose $\delta \leq \kappa$ is inaccessible and let $S_{\delta}=\{X \in$ $P_{\omega_{1}}\left(V_{\delta}\right): X<V_{\delta}$ and $\left(\mathbb{R}^{*}\right)^{N[g]} \subset \mathbb{R}^{*}$ for any $g$ such that for some $\mathbb{P} \in N, g \subset \mathbb{P}$ and $g$ is $N$-generic for $\mathbb{P}$, where $N=$ coll $(X)=$ transitive collapse of $X\}$. If $\delta<\kappa$ I claim $S_{\delta}$ contains a set closed and unbounded in $P_{\omega_{1}}\left(V_{\delta}\right)$. If not then $a_{\delta}=$ $P_{\omega_{1}}\left(V_{\delta}\right) \backslash S_{\delta}$ is stationary in $P_{\omega_{1}}\left(V_{\delta}\right)$. Let $G \subset \mathbb{Q}_{<\kappa}$ be $V$-generic with $a_{\delta} \in G$ and let $j: V \rightarrow M \subset V[G]$ be the induced embedding. Thus $j^{\prime \prime}\left(\cup a_{\delta}\right) \in j\left(a_{\delta}\right)$. However, $\cup a_{\delta}=V_{\delta}$ and so $j^{\prime \prime}\left(V_{\delta}\right) \in j\left(a_{\delta}\right)$. But $V_{\delta}=\operatorname{coll}\left(j^{\prime \prime}\left(V_{\delta}\right)\right)$; hence, for some $\mathbb{P} \in$ $V_{\delta}$ and $g \in M, g \subset \mathbb{P}, g$ is $V_{\delta}$-generic and $\left(\mathbb{R}^{*}\right)^{V_{\delta}[g]} \not \subset\left(\mathbb{R}^{*}\right)^{M}$. $\delta$ is inaccessible so $g$ is $V$-generic for $\mathbb{P}$ and $\left(\mathbb{R}^{*}\right)^{V_{\delta}[g]}=$ $\left(\mathbb{R}^{*}\right)^{V[s]}$. Further $\left(\mathbb{R}^{*}\right)^{M}=\left(\mathbb{R}^{*}\right)^{V[G]}$. But by Theorem 6 , $\left(\mathbb{R}^{*}\right)^{V[g]} \subset\left(\mathbb{R}^{*}\right)^{V[G]}$, a contradiction. Hence for each inaccessible $\delta<\kappa, S_{\delta}$ contains a set closed and unbounded in $P_{\omega_{1}}\left(V_{\delta}\right) . \kappa$ is supercompact; hence, by reflection $S_{\kappa}$ contains a set closed and unbounded in $P_{\omega_{1}}\left(V_{k}\right)$. Fix $F_{0}: V_{\kappa}^{<\omega} \rightarrow V_{\kappa}$ such that $\left\{X \in P_{\omega_{1}}\left(V_{\kappa}\right): F_{0}^{\prime \prime}\left(X^{<\omega}\right) \subset X\right\} \subset S_{\kappa}$. For $h \in(\omega \times$ $\omega)^{\omega}$ let $E_{h}=\operatorname{range}(h)$. Let $A=\left\{(x, h): x \in \omega^{\omega}, h \in(\omega \times \omega)^{\omega}\right.$, $\left(\omega, \mathrm{E}_{\mathrm{h}}\right) \vDash \mathrm{ZFC}, E_{h}$ is well-founded, and if $N=\operatorname{coll}\left(\omega, E_{h}\right)$ then for some $\mathbb{P} \in N$ and for some $g \subset \mathbb{P}, g$ is $N$-generic for $\mathbb{P}$ and $\left.x \in\left(\mathbb{R}^{*}\right)^{N[g]}\right\}$. One can show that $A$ is $\Pi_{1}^{1}$. Fix a $\Pi_{1}$ formula $\varphi\left(t_{1} t_{2}\right)$ such that $A=\left\{(x, h) \mid V_{\omega+1} \vDash \varphi[x, h]\right\}$. Choose a tree $T_{0}$ on $(\omega \times \omega) \times \kappa$ such that for any $\mathbb{P} \in V_{\kappa}$, if $G \subset \mathbb{P}$ is $V$-generic then $V[G] \vDash " p\left[T_{0}\right]=\left\{(x, h) \mid(V[G])_{\omega+1}\right.$ $=\varphi[x, h]\}$." Fix an enumeration $\left\langle s_{k}: k \in \omega\right\rangle$ of $\omega^{<\omega}$ such that for each $k \in \omega$, dom $s_{k} \cup$ rng $s_{k} \subset k$. Define $T$ as a tree on $\omega$ $\times(\omega \times \omega) \times \kappa \times V_{\kappa}$ such that $(x, h, g, f) \in[T]$ iff $(i)(x, h, g)$ $\in\left[T_{0}\right],(i i) E_{h}=\{(i, j): f(i) \in f(j)\},(i i i)$ for each $i \in \omega, E_{h} \cap i$ $\times i \subset \operatorname{range}\left(h\left\lceil i^{2}\right)\right.$, and (iv) for each $k \in \omega, f(2 k+1)=F_{0}(f \circ$ $\left.s_{k}\right)$. Similarly define $T^{*}$ from $A^{*}=\left\{(x, h): x \in \omega^{\omega}, \ldots\right.$ and $x$ $\left.\notin\left(\mathbb{R}^{*}\right)^{N[8]}\right\}$. Thus $p[T]=\mathbb{R}^{*}$ and $p\left[T^{*}\right]=\omega^{\omega} \backslash \mathbb{R}^{*}=$ $\omega^{\omega} \backslash p[T]$. Finally for any $\mathbb{P} \in V_{k}$, if $G \subset \mathbb{P}$ is $V$-generic then

$$
V[G] \vDash " \mathbb{R}^{*} \subset p[T] "
$$

and

$$
V[G] \vDash "\left(\omega^{\omega} \backslash \mathbb{R}^{*}\right) \in p\left[T^{*}\right] . "
$$

By absoluteness

$$
V[G] \vDash " p[T] \cap p\left[T^{*}\right]=\emptyset "
$$

and so

$$
V[G] \vDash " p[T]=\mathbb{P}^{*} \text { and } p\left[T^{*}\right]=\omega^{\omega} \backslash p[T] . "
$$

THEOREM 8. Suppose $\kappa$ is supercompact. Suppose $\mathrm{T}$ and $T^{*}$ are trees on $\omega \times \kappa$ such that for any partial order $\mathbb{P} \in \mathrm{V}_{\kappa}$, if $\mathrm{G} \subset \mathbb{P}$ is $\mathrm{V}$-generic then $\mathrm{V}[\mathrm{G}] \vDash " \mathrm{p}\left[\mathrm{T}^{*}\right]=\omega^{\omega} \backslash \mathrm{p}[\mathrm{T}]$." Then $\mathrm{T}$ and $\mathrm{T}^{*}$ are each $\kappa$-weakly homogeneous.

Proof: Let $m_{\kappa}\left(\kappa^{<\omega}\right) \subset m\left(\kappa^{<\omega}\right)$ be the set of $\kappa$-complete measures on $\kappa^{<\omega}$. Choose $\tau<V_{\kappa+2},|\tau|<\kappa$, with $\left\{T, T^{*}\right\} \subset \tau$ such that $(i)$ if $\nu \in m_{\kappa}\left(\kappa^{<\omega}\right)$ then $\nu \cap \tau=\mu \cap \tau$ for some (necessarily unique) $\mu \in \tau$ and (ii) if $\left\langle\mu_{k}: k \in \omega\right\rangle$ is a tower of measures in $\tau \cap m_{\kappa}\left(\kappa^{<\omega}\right)$ then the tower is countably complete iff for some $f \in \kappa^{\omega}, f \nmid k \in \cap\left\{A \in \tau: \mu_{k}(A)=1\right\}$ for all $k \in \omega$. To see that such a set $\tau$ exists, consider in $M$ the set $j^{\prime \prime}\left(V_{\kappa+2}\right)<j\left(V_{\kappa+2}\right)$, where $j: V \rightarrow M$ is an elementary embedding, $\operatorname{cp}(j)=\kappa$ and $M^{V_{k+2}} \subset M$. For $s \in \kappa^{<\omega}$ let $\mu(\tau, s)$ denote that measure $\mu \in \tau$ such that $\mu \cap \tau=\left\{A \in \tau: A \subset \kappa^{<\omega}\right.$ and $s \in A\}$. Note that for any $f \in \kappa^{\omega},\langle\mu(\tau, f \mid k): k \in \omega\rangle$ is a countably complete tower.

Fix $\kappa_{0}<\kappa,|\tau|<\kappa_{0}$, such that $\kappa_{0}$ is $V_{\kappa_{0}+1}$-supercompact. Let $G_{0} \subset \mathbb{Q}_{<\kappa_{0}}$ be $V$-generic and let $j_{0}: V \rightarrow M_{0} \subset V\left[G_{0}\right]$ be the induced embedding. Since $|\tau|<\kappa_{0}, j_{0}^{\prime \prime}(\tau) \in M_{0}$ and $M_{0} \vDash$ " $j_{0}^{\prime \prime}(\tau)$ is countable." I shall show that $M_{0} \vDash$ "for each $x_{0} \in$ $p\left[j_{0}(T)\right]$ there exists a countably complete tower $\left\langle\nu_{k}: k \in \omega\right\rangle$ of $j_{0}(\kappa)$-complete measures in $j_{0}^{\prime \prime}(\tau)$ such that for each $k \in \omega$, $\nu_{k}\left(j_{0}(T)_{x_{0}}\right)=1$." Given this it follows that $T$ is $\kappa$-weakly homogeneous. We work in $V\left[G_{0}\right]$. Fix $x_{0} \in M_{0}$ such that $x_{0} \in$ $p\left[j_{0}(T)\right]$. Clearly $p[T] \subset p\left[j_{0}(T)\right]$ and $p\left[T^{*}\right] \subset p\left[j_{0}\left(T^{*}\right)\right]$. But $p\left[T^{*}\right]=\omega^{\omega} \backslash p[T]$ since $\mathbb{Q}_{<\kappa_{0}} \in V_{\kappa}$. Hence $p[T]=p\left[j_{0}(T)\right]$ and so $x_{0} \in p[T]$. Choose $f_{0} \in \kappa^{\omega}$ such that $\left(x_{0}, f_{0}\right) \in[T]$. For each $k \in \omega$ let $\mu_{k}=\mu\left(\tau, f_{0} \mid k\right)$ and so $\mu_{k} \in V$ and $V \vDash$ " $\mu_{k}$ is a $\kappa$-complete measure." Define $\bar{f}_{0}$ by $\bar{f}_{0}(k)=$ $j_{0}\left(f_{0}(k)\right) . M_{0}^{\omega} \subset M_{0}$ in $V\left[G_{0}\right]$, hence $\bar{f}_{0} \in M_{0}$ and $\left\langle j_{0}\left(\mu_{k}\right): k \in\right.$ $\omega\rangle \in M_{0}$. Clearly $M_{0} \vDash " j_{0}\left(\mu_{\kappa}\right)\left(j_{0}(T)_{x_{0}}\right)=1$ for all $k \in \omega "$ and $M_{0} \vDash "\left\langle j_{0}\left(\mu_{k}\right): k \in \omega\right\rangle$ is a tower of measures." Thus it suffices to show $M_{0} \vDash$ "the tower $\left\langle j_{0}\left(\mu_{k}\right): \kappa \in \omega\right\rangle$ is countably complete." But this is immediate since $M_{0} \vDash$ "for each $k \in \omega, j_{0}\left(\mu_{k}\right)=\mu\left(j_{0}(\tau), \bar{f}_{0}\lceil k) . "\right.$

A similar argument shows that $T^{*}$ is $\kappa$-weakly homogeneous.

Theorem 1 now follows easily. Suppose $\kappa$ is supercompact. By Theorems 7 and $8, \mathbb{R}^{*}$ is the projection of a $\kappa$-weakly homogeneous tree. Every set of reals, $A$, with $A \in L(\mathbb{R})$ is continuously reducible to $\mathbb{R}^{*}$. Therefore each such set is the projection of a $\kappa$-weakly homogeneous tree. 
Remarks: All of the theorems here can be proved from substantially weaker large cardinal assumptions. Further, the potential influence is well beyond the sets of reals in $L(\mathbb{R})$. See, for example, the following theorem.

THEOREM. Assume $\kappa$ is supercompact. Assume the continuum hypothesis. Then every $\Sigma_{1}^{2}$-definable set of reals is the projection of a $\kappa$-weakly homogeneous tree.

These related results and results for $\mathbb{P}_{<\kappa}$ will be published in a forthcoming paper on large cardinals and determinacy.

The assertion that every set of reals, in $L(\mathbb{R})$, is the projection of a weakly homogeneous tree has consequences beyond the usual regularity properties such as Lebesgue measurability. For example by results of Kechris it follows that $L(\mathbb{R}) \vDash$ " $\omega_{1}$ is measurable." It may even be that this alone implies $\mathrm{L}(\mathbb{R}) \vDash A D$.

Question: Suppose every set of reals that belongs to $L(\mathbb{R})$ is the projection of a weakly homogeneous tree. Does $A D$ hold in $L(\mathbb{R})$ ?

The main question left open here is whether the existence of a supercompact cardinal implies $L(\mathbb{R}) \vDash A D$. If so, this would be a dramatic reduction in the large cardinal hypothe- sis sufficient to prove $\mathrm{AD}^{L(R)}$. Martin and Steel (6) have answered this question. They show, among other things, that if $\kappa$ is supercompact then the projection of every $\kappa$-weakly homogeneous tree is determined and so by Theorem $1, L(\mathbb{R}) \vDash$ AD.

This research was partially supported by National Science Foundation Grant DMS 8416349. W.H.W. is an A. P. Sloan Foundation Fellow.

1. Jech, T. (1978) Set Theory (Academic, New York), p. 548.

2. Martin, D. A. (1970) Fund. Math. 66, 287-291.

3. Foreman, M. D., Magidor, M. \& Shelah, S. (1988) Ann. Math. 127, 1-47.

4. Kechris, A. S. (1981) in Cabal Seminar 77-79, Lecture Notes in Mathematics, eds. Kechris, A. S., Martin, D. A. \& Moschovakis, Y. N. (Springer, Berlin), pp. 62-66.

5. Solovay, R. M. (1978) in Cabal Seminar 76-77, Lecture Notes in Mathematics, eds. Kechris, A. S., Martin, D. A. \& Moschovakis, Y. N. (Springer, Berlin), pp. 178-181.

6. Martin, D. A. \& Steel, J. R. (1988) Proc. Natl. Acad. Sci USA $85,6582-6586$. 